\title{
Affective Learning with an EEG Approach
}

\author{
$\mathrm{Bin} \mathrm{Hu}$ \\ School of Information Science and Engineering \\ Lanzhou University, China, and \\ Department of Computing, Birmingham City University, UK \\ binhu@lzu.edu.cn
}

People's moods heavily influence their way of communicating and their acting and productivity, which also plays a crucial role in learning process. Affective learning is an important aspect of education. Emotions of learners need to be recognized and interpreted so as to motivate learners and deepen their learning, and this is a prerequisite in e-learning. Normally, affective learning has been investigating some technologies to understand learners' emotions through detecting their face, voice and eyes motion, etc.

Our research focuses on how to enhance interactive learning between learners and tutors, and understand learners' emotions through an EEG approach. We have developed an e-learning environment and recorded EEG signals of learners while they are surfing in the website. We presented an ontology based model for analyzing learners' alpha wave (a component of EEG signals) to infer the meaning of its representation, then to understand learners' emotions in learning process. The outcomes of the research can contribute to evaluation of e-learning systems and deepen understanding of learners' emotions in learning process. 